\title{
B-cell responses to vaccination at the extremes of age
}

\section{Claire-Anne Siegrist* and Richard Aspinall ${ }^{\ddagger}$}

Abstract | Infants and the elderly share a high vulnerability to infections and therefore have specific immunization requirements. Inducing potent and sustained B-cell responses is as challenging in infants as it is in older subjects. Several mechanisms to explain the decreased B-cell responses at the extremes of age apply to both infants and the elderly. These include intrinsic B-cell limitations as well as numerous microenvironmental factors in lymphoid organs and the bone marrow. This Review describes the mechanisms that shape B-cell responses at the extremes of age and how they could be taken into account to design more effective immunization strategies for these high-risk age groups.

\section{Infant \\ A $\leq 12$-month-old human baby or a $\leq 3$-week-old mouse (experimental definition) \\ Neonate \\ A $\leq$ 28-day-old human infant or a $\leq 7$-day-old mouse.}

*WHO Collaborative Center for Neonatal Vaccinology. Departments of Pathology Immunology and Pediatrics, Medical Faculty of the University of Geneva, Centre Medical Universitaire, 1 rue Michel Servet, 1211 Geneva 4, Switzerland. ${ }^{\ddagger}$ Translational Medicine, Cranfield Health, Vincent Building, Cranfield University, Bedfordshire MK43 OAL, UK. Correspondence to C.-A.S. e-mail: claire-anne.siegrist@unige.ch doi:10.1038/nri2508
Respiratory and diarrhoeal diseases caused by some pathogens - respiratory syncytial virus (RSV), measles virus, Bordetella pertussis, Streptococcus pneumoniae, Haemophilus influenzae type b (Hib), rotavirus or Salmonella spp. - claim the lives of more than 1.6 million young children each year ${ }^{1}$, and the global infant mortality rate still amounts to 49 deaths per 1,000 live births (see the World Health Organization (WHO) health statistics website). The abrupt transition at birth from a sterile intra-uterine environment to a foreign environment places neonates in a challenging situation. Innate immune responses can be readily elicited at birth, although with specific characteristics (reviewed in REF. 2), but they are transient and insufficient for immune protection. Furthermore, early immune protection initially relies on IgG antibodies of maternal origin ${ }^{3}$, the levels of which decline after birth with a half-life of 21-30 days. Therefore, infants would become vulnerable to infections within a short time frame without the development of adaptive immunity to confer sustained protection. As adaptive immunity in infants is readily amenable to enhancement through vaccination, great efforts and resources have been dedicated to infant immunization, which has had a notable impact on morbidity and mortality. However, early-life immune responses are weaker and of shorter duration than those that are elicited in immunologically mature hosts. Consequently, vaccine efficacy in this vulnerable population is limited.

This susceptibility to infection at the start of life is mirrored in those who approach the end of the normal life expectancy curve. Indeed, the elderly not only have problems in dealing with new pathogens but also have difficulties in responding to pathogens that they have previously overcome, from which they can suffer increasingly severe symptoms. Numerous infections have a higher incidence in the elderly and have a higher mortality rate than in younger adults ${ }^{4,5}$. For example, in the United States, the yearly return of influenza and RSV infections between 1990 and 1999 was responsible for 51,203 and 17,358 deaths, respectively ${ }^{6}$. In an average year, influenza, invasive pneumococcal disease and hepatitis B account for more than 50,000 deaths in the United States, with older individuals being 1,000 times more likely to die of these vaccine-preventable diseases than children living in developed countries ${ }^{7}$.

Current vaccines mediate their protective efficacy through the generation of neutralizing antibodies. Decades of studies have shown that antigen exposure in early life results in blunted, delayed or undetectable antibody responses to infections and immunizations. Historically, the neonatal immune system has been considered to be poorly competent in generating immune responses and instead to be polarized towards the induction of immune tolerance ${ }^{8}$. A conceptual switch occurred in the late 1990s through numerous demonstrations that adult-like B- and T-cell responses could be achieved in early life under specific conditions of stimulation (reviewed in REFS 9,10). However, as early-life antibody responses markedly differ from those elicited in mature hosts, great challenges remain to be encountered during vaccine development. At the other extreme of age, the capacity to induce protective antibody at a sufficient titre to prevent infection declines significantly with age: in individuals of 65 years or older, influenza ${ }^{11}$ and hepatitis $\mathrm{B}^{12}$ vaccines induce protective antibody titres in less than half of their recipients. 


\begin{tabular}{|c|c|c|}
\hline Cell type or site & Characteristics in infants & Characteristics in the elderly \\
\hline Naive B cells & $\begin{array}{l}\text { Decreased expression of cell-surface receptors } \\
\text { (CD21, CD40, CD80 and CD86) }\end{array}$ & Limited generation of naive B cells \\
\hline \multirow[t]{3}{*}{ Plasma cells } & $\begin{array}{l}\text { Limited lgG responses to protein antigen under } \\
12 \text { months of age }\end{array}$ & Poor lgG responses to protein antigen \\
\hline & $\begin{array}{l}\text { Limited IgG responses to most polysaccharide } \\
\text { antigens under } 18-24 \text { months of age }\end{array}$ & $\begin{array}{l}\text { Poor lgG responses to most polysaccharide } \\
\text { antigens }\end{array}$ \\
\hline & $\begin{array}{l}\text { Limited persistence of } \lg G \text { antibodies under } \\
12 \text { months of age }\end{array}$ & Decreased persistence of $\lg G$ antibodies \\
\hline \multirow[t]{3}{*}{ Memory B cells } & $\begin{array}{l}\text { Effective priming of memory B cells at and } \\
\text { before birth }\end{array}$ & $\begin{array}{l}\text { Accumulation of memory B cells of restricted } \\
\text { diversity }\end{array}$ \\
\hline & Progressive diversified lgG repertoire & Limited diversified lgG repertoire \\
\hline & $\begin{array}{l}\text { Limited affinity maturation under } 4-6 \text { months } \\
\text { of age }\end{array}$ & Affinity maturation is not affected by age \\
\hline \multirow[t]{2}{*}{ Germinal centres } & Impaired germinal centre responses ${ }^{\star}$ & Impaired germinal centre responses* \\
\hline & Limited functional FDC network* & Limited functional FDC network* \\
\hline Bone marrow & Limited access to plasma cell niches* & Limited access to plasma cell niches* \\
\hline
\end{tabular}

Responses are compared with healthy adults. *Shown in mice. FDC, follicular dendritic cell.

In this Review, we discuss the mechanisms that could account for the limitations of B-cell responses at the extremes of age, which are both interesting biological phenomena and key considerations for the design of more effective immunization strategies.

\section{B-cell responses in early life}

Postnatal maturation of antibody responses. The vulnerability of children younger than 18-24 months of age to encapsulated bacteria such as pneumococcus, Hib and meningococcus has long been thought to reflect a general failure to generate T-cell-independent B-cell responses to most bacterial polysaccharides (reviewed in REF. 13). However, age also has a direct effect on the magnitude of antibody responses to T-cell-dependent protein antigens (TABLE 1). As an example, RSV infection only triggers limited T-cell-dependent B-cell responses in young infants, which has implications for the development of liveattenuated intranasal RSV vaccines ${ }^{14}$. Infant vaccine studies worldwide have shown that there is an age-dependent stepwise increase in the rates of seroconversion and the magnitude of antibody responses, regardless of whether repeat immunization is administered ${ }^{15,16}$. In fact, the use of accelerated schedules in which immunizations are initiated at 6-8 weeks of age and repeated at 4-week intervals increases the proportion of infants who fail to respond to certain immunogens $s^{17,18}$ and might decrease protective efficacy against many important infant pathogens, such as pertussis ${ }^{1,19}$. Direct evidence of the postnatal maturation of antibody responses was provided by single-dose studies with measles-mumps-rubella ${ }^{20,21}$ and hepatitis $\mathrm{A}^{22}$ vaccines. The IgG responses that can be eventually elicited in infants by several doses of vaccine wane rapidly, such that most children have low or undetectable antibody concentrations 6-9 months after their primary infant immunization series ${ }^{18,23}$. This can result in a resurgence of vulnerability to infection unless repeat antigen exposure occurs after the first year of life ${ }^{24,25}$.
Induction of a primary response. The mechanisms that shape B-cell responses in early life were identified using neonatal mouse immunization models that were developed to reproduce the main limitations of immune responses to vaccines that are administered at an early age (reviewed in REFS 9,26) (FIG. 1). There are numerous differences (TABLE 1) between neonatal and adult mouse splenic B cells (reviewed in REF. 10), although fewer differences have been identified by comparing human peripheral B cells. Specifically, human neonatal B cells express lower levels of the co-stimulatory molecules $\underline{\mathrm{CD} 40}, \underline{\mathrm{CD} 80}$ and $\underline{\mathrm{CD} 86}$, which decreases their responses to CD40 ligand (CD40L) and interleukin-10 (IL-10) ${ }^{27}$ expressed by T cells. Splenic marginal zone infant B cells express lower levels of CD21 (REF. 28), which limits their capacity to respond to polysaccharide-complement complexes $^{29}$. The expression of TACI (transmembrane activator and calcium-modulating cyclophilin-ligand interactor; also known as TNFRSF13B), an important co-stimulatory receptor, is also decreased on both neonatal mouse $\mathrm{e}^{30}$ and neonatal human B cells, particularly those born prematurely ${ }^{27}$.

In addition, B-cell responses in early life are influenced by numerous extrinsic factors (TABLE 1). Antibodies of maternal origin bind to vaccine antigens in an epitopespecific manner and therefore prevent infant B cells from accessing immunodominant vaccine epitopes ${ }^{31}$. Furthermore, human ${ }^{32}$ and mouse ${ }^{33}$ neonates have low levels of serum complement component C3, which limits their responses to antigen-C3d complexes. The human spleen contains fewer marginal zone macrophages (which have a crucial role in the induction of an antibody response through the trapping of particulate antigens) in neonates than in adults ${ }^{28}$, and the cells differ in their capacity to produce cytokines ${ }^{34}$. In infant mice, B-cell responses are limited by a marked delay in the maturation of the follicular dendritic cell (FDC) network, resulting from the failure of FDC precursors 
A

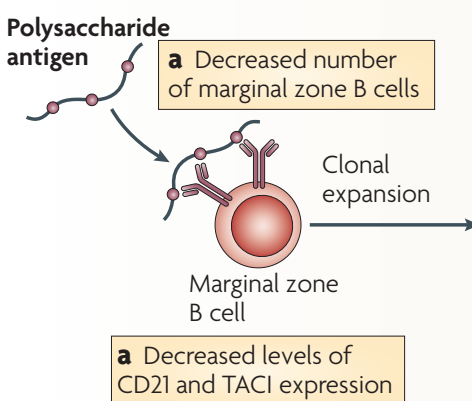

Spleen or lymph node

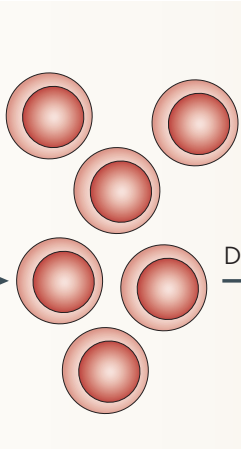

Blood

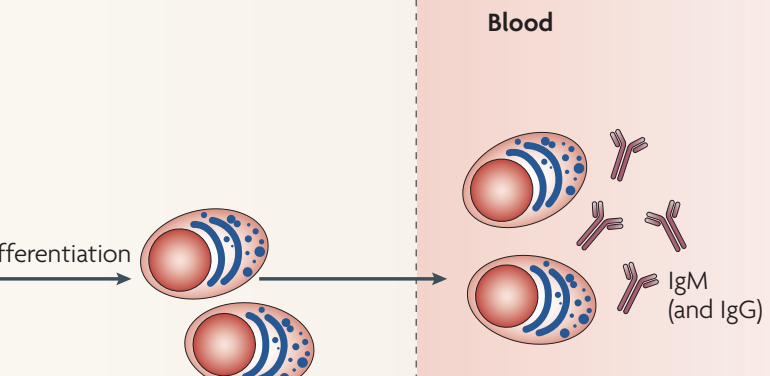

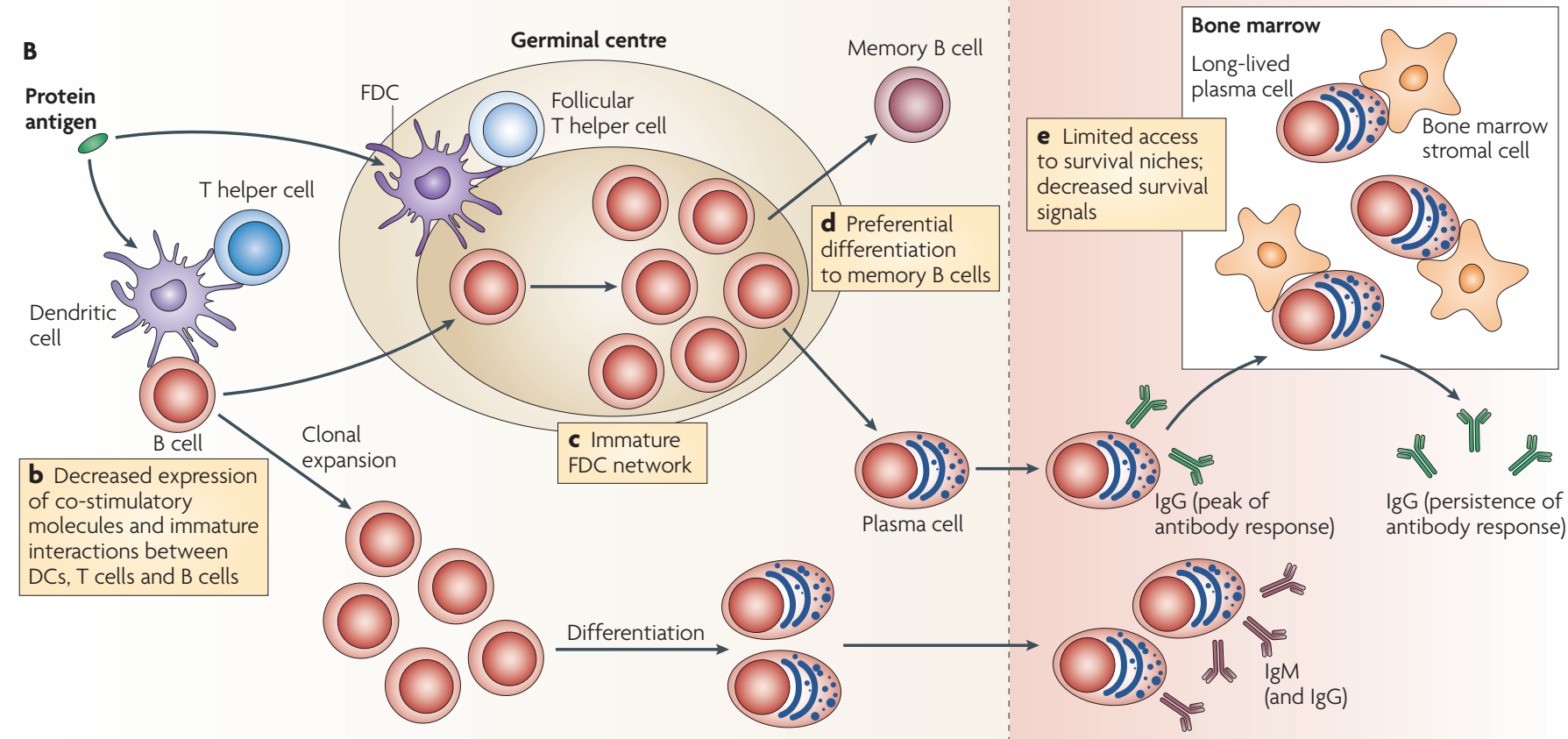

Figure 1 | Early-life limitations of B-cell responses. A | Polysaccharides reach the marginal zone of lymphoid organs, bind to marginal zone B cells and drive their differentiation to short-lived plasma cells. In early life, a decreased number of marginal zone B cells and decreased levels of expression of CD21 and/or TACI (transmembrane activator and calciummodulating cyclophilin-ligand interactor) limit the activation capacity of B cells (a), resulting in the generation of fewer plasma cells. B | Proteins reach the B-cell zone in lymphoid organs through free fluid diffusion, triggering T-cellindependent responses, and/or reach the T-cell zone through dendritic cell (DC) transport. Early-life B-cell activation is limited by the decreased expression of co-stimulatory receptors (CD40, CD80 and CD86) and by immature B-cell-DC-T-cell interactions (b). This affects extrafollicular and germinal centre responses. Activated B cells, attracted by antigen-bearing follicular DCs (FDCs) that nucleate germinal centre reactions, receive help from follicular Thelper cells, proliferate, undergo somatic hypermutation and affinity maturation, and switch from lgM- to lgG-, $\lg \mathrm{A}$ - or $\lg \mathrm{E}$-producing cells. The immaturity of the FDC network (c), or of other crucial germinal centre factors, limits early-life germinal centre responses. Germinal centre B cells differentiate into memory B cells or plasma cells. In early life, there is a preferential differentiation towards memory B cells (d). By contrast, fewer plasma cells are elicited, resulting in lower peak lgG titres. Short-lived plasma cells must access survival niches that are located in the bone marrow to differentiate into long-lived plasma cells. The decreased persistence of early-life lgG responses might result from limitations of access to bone marrow niches and/or their failure to provide appropriate survival signals (e), such as a proliferation-inducing ligand (APRIL).

to respond to B-cell-mediated lymphotoxin-a signalling ${ }^{35}$. FDCs nucleate germinal centre reactions by attracting antigen-specific B cells, retain antigens in the form of immune complexes that are highly stimulatory to B cells and provide signals that lead to somatic hypermutation and class-switch recombination. The immaturity of the FDC network therefore delays the induction and limits the magnitude of germinal centre responses, even when potent adjuvants that induce adult-like B-cell, T-cell and DC activation patterns are used $^{35}$ (C.-A.S., unpublished observations). It is difficult to establish whether functional FDC maturation is delayed in humans. However, the slow increase in the size and number of lymph node germinal centres with age $^{36}$ and the induction of fewer plasma cells following infant immunization, compared with adult immunization, indicate that human infants have limited germinal centre reactions ${ }^{37}$. 
Moreover, immature DC-T-cell interactions might also limit infant IgG responses. In addition, neonatal $\mathrm{CD}^{+} \mathrm{T}$-cell responses differ from those elicited later in life, showing preferential T-helper-2-cell polarization (reviewed in REF. 10). This could either support or limit neonatal antibody responses, depending on the antigenspecific B-cell requirements for co-stimulation. It is unclear whether infant $\mathrm{T}$ cells express sufficient CD40L to provide optimal CD40-mediated co-stimulation to infant B cells. Potent adjuvants (such as CpG oligonucleotides in mice) can induce adult-like DC and T-cell activation patterns, but they fail to induce adult-like antibody responses ${ }^{35,38}$. This highlights the control that is exerted by microenvironmental determinants such as FDCs on B-cell responses in early life. All of these factors (TABLE 1) have an age-dependent influence on immune responses by limiting the kinetics and magnitude of antibody responses to both polysaccharide and protein antigens in early life.

Antibody persistence. The long-term maintenance of specific antibodies with a short half-life requires the persistence of antibody-producing B cells, which can either be continually produced from a pool of memory B cells or can persist as long-lived plasma cells. Antibodymediated depletion of memory B cells, which does not affect plasma cells, has shown that the plasma-cell stage is independent of the memory B-cell pool ${ }^{12}$. Indeed, the short persistence of mouse antibody responses in early life (TABLE 1) results from the failure to establish and maintain an optimal plasma-cell pool in the bone marrow $^{39}$. In infant mice, post-germinal-centre plasmablasts readily home towards the bone marrow compartment ${ }^{40}$ but fail to establish themselves as long-lived plasma cells because of insufficient differentiation and survival signals from bone marrow stromal cells ${ }^{40}$. This was recently ascribed to the insufficient production of a proliferation-inducing ligand (APRIL; also known as TNFSF13), which is a crucial factor for the establishment of the bone marrow plasma-cell pool by early-life bone marrow stromal cells ${ }^{41}$. Whether similar limitations affect the establishment and/or persistence of bone marrow plasma cells in early human life is not known. However, studies in human infant twins have shown that although the magnitude of primary vaccine responses is controlled by genetic determinants ${ }^{42}$, their persistence is influenced by environmental factors ${ }^{43}$. This supports our hypothesis that the limited persistence of antibody responses in early life results from exposure to a large load of environmental antigens, which results in competition for access to a limited set of plasma-cell survival niches in the bone marrow.

Priming and affinity maturation. Although neonatal antigen exposure rarely elicits marked antibody responses, it can effectively prime the immune system for enhanced subsequent responses ${ }^{44,45}$ (TABLE 1). This priming capacity was recently used to accelerate the development of antipertussis responses by administering a priming vaccine dose at birth, initially in mice ${ }^{26}$ and recently in humans ${ }^{46,47}$. Surprisingly, however, neonatal human immunization can sometimes fail to enhance subsequent antibody responses (for example, for tetanus or diphtheria toxoids ${ }^{48}$ ) or even impair such responses (for example, for pertussis antigens $\left.^{49}\right)$. Such vaccine interference could result from B-cell competition for limited resources (such as co-stimulatory signals), from changes in T-cell polarization, from the active induction of regulatory $\mathrm{T}$ cells or from as yet unidentified mechanisms $\mathrm{s}^{50}$.

The acquisition of full antigen responsiveness also requires a progressive diversification of the IgG antibody repertoire. Earlier studies reported a delayed acquisition of somatic mutations in the $\operatorname{IgG~} \mathrm{V}_{\mathrm{H}}$ (variable region of the immunoglobulin heavy chain) ${ }^{51}$ and $\mathrm{V}_{\mathrm{L}}$ (variable region of the immunoglobulin light chain) regions during infant antibody responses ${ }^{52}$, suggesting that there is limited affinity maturation of antibodies (TABLE 1). However, work in mice has shown that proteinbased human vaccines can induce adult-like avidity profiles in infants, in contrast to vaccines that are based on unique epitopes such as haptens $s^{53}$. Furthermore, affinity maturation has also been shown to occur in response to infant immunization with the Hib vaccine ${ }^{54}$, and the infant and adult rotavirus-specific B-cell repertoires are also highly related ${ }^{55}$.

The diversity of IgG transcripts and somatic mutation frequency increase within the first months of life ${ }^{56}$, and adult levels of somatic mutation were reported at 8 months of age in unselected peripheral blood $\mathrm{B}$ cells ${ }^{51}$. So, age-related differences in the immunoglobulin repertoire and variance of B-cell responses to immunization seem to arise from selection rather than from changes in recombination of the immunoglobulin locus itself ${ }^{57}$. Nevertheless, the avidity of measles-specific IgG is significantly weaker when elicited in 6- or 9-month-old infants than in 12-month-old infants ${ }^{58}$. Therefore, neonatal immunization does prime antigen-specific $\mathrm{B}$ and $\mathrm{T}$ cells, but this priming can accelerate, leave unchanged or impair subsequent antibody responses and affinity maturation. The determinants of this process are unknown, and identifying them will require further studies.

Determinants of memory responses. The administration of a single dose of vaccine at birth can fail to elicit specific antibodies while priming for subsequent secondary responses, which indicates a preferential neonatal differentiation pathway towards memory B cells rather than plasma cells. Several factors seem to contribute to this B-cell differentiation pattern (BOX 1). The fate of antigen-specific naive $B$ cells and their differentiation to short-lived plasma cells, long-lived plasma cells or memory B cells is controlled by early B-cell activation signals. High-affinity B cells are actively recruited to the plasma-cell pool, whereas moderate-affinity B cells remain as memory $B$ cells in the secondary lymphoid organs ${ }^{59}$. So, decreased initial B-cell receptor affinity and/or delayed affinity maturation of neonatal naive $B$ cells might decrease the strength of the signal and favour memory B-cell differentiation. The limited expression of CD21 by infant B cells also supports the generation of memory B cells and impairs the development of plasma cells ${ }^{60}$, which would be supported 


\section{Box 1 |Favouring memory over antibody responses: evolutionary adaptation?}

Mechanisms that favour memory B-cell rather than plasma-cell differentiation:

- Decreased strength of B-cell receptor (BCR) signalling by naive B cells

- Decreased available antigen as a result of binding by maternal antibodies

- Limited expression of B-cell co-stimulatory receptors (CD21, CD40, CD80 and CD86)

- Limited co-stimulatory signals to B cells (through CD40 ligand, serum complement component $\mathrm{C} 3$, cytokines, B-cell-activating factor (BAFF) and/or a proliferationinducing ligand (APRIL))

- Delayed follicular dendritic cell (FDC) maturation, which limits germinal centre reactions

- B-cell competition for simultaneous access to limited germinal centre resources

- Limited support for the establishment of bone marrow plasmablasts

- Intense exposure to environmental antigens, which results in B-cell competition for simultaneous access to limited bone marrow survival niches

Potential evolutionary factors that limit plasma-cell differentiation in early life:

- Antibodies of maternal origin decrease the antigen load and provide protection, so there is no drive for plasma-cell differentiation

- Preventing plasma-cell differentiation until memory B cells have been primed and/or reactivated could limit potentially harmful cross-reactive responses to self polysaccharides and glycosylated proteins during the vulnerable periods of fetal and postnatal development

- As a mechanism to limit responses against prevalent but harmless environmental antigens (resource sparing)

- As a mechanism to prepare the post-weaning period for prompt reactivation in case of repeat exposure evidence that its magnitude or persistence is similar to that elicited in immunologically mature hosts. Recent observations of the failure of booster vaccines to elicit a memory response in adolescents or young adults that had been primed against hepatitis B virus in infancy suggest that infant-triggered memory B cells might not be life long ${ }^{66,67}$. Whether this reflects a smaller B-cell pool in infants and/or the influence of as yet undefined homeostatic factors remains to be determined.

In summary, a large number of B-cell intrinsic factors and extrinsic determinants cooperate to limit the induction and the persistence of antibody-secreting plasma cells in early life, while supporting the preferential induction of memory B-cell responses.

\section{B-cell responses in later life}

Antibody responses in adults and older individuals. Perhaps the greatest difference between B-cell responses at life's extremes regards their serological profiles. Successful recovery from viral and bacterial infections is associated with good humoral immune responses, as shown by the increased levels of specific antibodies following infection. Surviving to old age requires a successful response to a wide range of potential pathogens and the generation of a prodigious 'reservoir' of immunological memory. Measurable titres of serum antibody are stable and have the capacity to be maintained for prolonged periods. Antibody responses to viruses, such as varicella-zoster, measles and mumps, have half-lives of 50 years or more, whereas antibody responses to non-replicating protein antigens, such as tetanus and diphtheria toxins, decline with half-lives of 10-20 years ${ }^{68}$. This suggests that the persistence of antigen contributes to the continued production of antibody, as indicated by studies in mice ${ }^{69}$.

The capacity to respond to new potential pathogens does not increase at an exponential rate throughout life. Indeed, both extremes of life are linked by an inability to respond to new antigens, albeit for mechanistically different reasons (TABLE 1). Evidence for this in older individuals comes from the measurement of changes in specific antibody levels at defined times after vaccination. The ability of influenza vaccine to induce protection is related to age, with an efficacy between $70 \%$ and $90 \%$ in those under 65 years of age, but of $30 \%$ to $40 \%$ at best for those over 65 years of age $\mathrm{e}^{11,70}$. Similarly, responses to pneumococcal polysaccharides ${ }^{71}$ and hepatitis $\mathrm{B}$ vaccines are compromised by old age ${ }^{12}$, and antibody responses are of shorter duration in healthy older people ${ }^{72}$ (TABLE 1).

Epidemiological studies have shown that populations of older individuals have increasing titres of autoantibody as they age $\mathrm{e}^{73}$. These autoantibody titres are not usually associated with autoimmune disease, possibly because of the low affinity of the antibodies. More than half of healthy older individuals have antibodies for non-organspecific autoantigens (such as nucleoprotein or $\operatorname{IgG})^{74}$, which might result from their generation as a by-product of responses to other antigens. Indeed, an early study showed that shortly after vaccination with tetanus toxoid, there was a significant increase in the frequency of B cells producing rheumatoid factor, accompanied by an increase in plasma levels of IgM rheumatoid factor ${ }^{75}$. There is also
An autoantibody specific for the Fc portion of $\lg \mathrm{G}$, which is most relevant in rheumatoid arthritis. by CD40-mediated signalling, cytokines such as IL-21 activating factor (BAFF; also known as TNFSF13B) and $\mathrm{APRIL}^{62}$. Remarkably, these plasma-cell-supporting facunless additional activation signals are provided to Furthermore, early-life B cells might have to compete for limited resources within the germinal centre, which impairs antibody responses. Indeed, plasma-cell additional DC activation signals. For example, Hib va gated to the meningococcal outer membrane protein complex (PRP-OMP vaccine), which induces Toll-like( $\mathrm{Hib}$ conjugate vaccines ${ }^{64}$. However, this is at the expense of the memory B-cell pool, as PRP-OMP-induced B cells viding three early infant doses of potent vaccines, such as serogroup C meningococcus-tetanus conjugates, forces primary antibody responses to the detriment of memory B-cell responses ${ }^{65}$.

Therefore, a combination of factors results in the preferential differentiation of early-life B cells towards memory B cells instead of long-lived plasma cells in a pattern that is so strikingly conserved (at least between mice and humans) that it suggests to us an evolutionary adaptation (BOX 1). Importantly, although it has been shown that the memory B-cell pool can be formed early in life, this should not be considered as 
an age-dependent increase in the prevalence of organspecific antibodies (such as thyroid-specific antibodies $)^{76}$. The increase does not seem to be exponential, as studies showed that the prevalence of organ-specific autoantibodies in centenarians is similar to that found in individuals under 50 years of age $\mathrm{g}^{76}$.

Further changes in the serological profile in older individuals were revealed by the presence of a single spike on electrophoresis gels within the region of the gel that is associated with immunoglobulins. These paraproteins are produced by a clone of plasma cells with a defined single specificity - a condition known as benign monoclonal gammopathy. The prevalence of this condition increases with age, with $3.2 \%$ of individuals over 50 years of age suffering from the disease, $5.3 \%$ of those over 70 years of age and $7.5 \%$ of those over 85 years of age $\mathrm{e}^{77}$.

The differences in the serum antibody profile between these age groups include the increase in the amount of serum immunoglobulin between neonates or infants and elderly individuals ${ }^{78}$, the increased presence of autoantibodies and low affinity antibodies in the elderly and the occurrence of an over-representation of specific classes of antibody from individual B-cell clones in some older individuals (TABLE 1). The following sections discuss how these changes are a reflection of changes in the B-cell pool.

Cellular profile. Comparison between the first and later years of life reveals considerable differences in the peripheral B-cell pool. Sites of naive B-cell production decrease with age: early in life most bones contain marrow that is actively producing $B$ cells, whereas in adults the haematopoietic bone marrow is restricted to the vertebral bones, sternum, ribs, flat bones of the cranium and pelvis, and the end of long bones. These active sites gradually decrease further, and fat deposits accumulate in the marrow cavity as a result of the differentiation of stromal cells to adipocyte-like cells ${ }^{79}$ (FIG. 2). These changes probably alter the specialized stromalcell microenvironment and result in the potential loss of specialized niches. The age-related decrease in the production of new B cells from their precursors, which has been described by many investigators, has indicated a decrease in the absolute numbers of early B-cell progenitors, including both pro-B and pre-B cells (reviewed in REF. 80). However, antigen encounter continues to induce immune responses and the proliferation of $\mathrm{B}$ cells throughout life. Therefore, with time, the average age of $B$ cells in the pool, as measured in terms of the number of cell divisions undertaken, increases because the contribution of new B cells from the bone marrow decreases but the absolute number of B cells remains similar.

The most marked difference between infants and elderly individuals is a progressive shift from a population that contains many naive B cells and few memory B cells to one that contains mostly memory $\mathrm{B}$ cells and few naive $\mathrm{B}$ cells in later years (FIG. 2). Subsets in the B-cell pool can be defined on the basis of their expression of CD10, CD19, CD23, CD27, CD38, CD44, CD77 and CD95 (REF. 81). Of these, CD27 has been used as a universal marker to define the memory B-cell subset ${ }^{81}$. However, the discovery of $\mathrm{CD} 27^{-}$memory $\mathrm{B}$ cells ${ }^{82}$ indicates that the memory B-cell pool is more heterogeneous than expected $^{83}$, which questions the use of this single marker as a method of identifying these cells. Analysis in humans is further hampered because a representative sample of the B-cell pool might not be provided by the peripheral blood. A prime example of this comes from responses to smallpox vaccination, which can be maintained for at least 70 years. Specifically, experiments on vacciniavirus-specific memory B cells in individuals that had been vaccinated more than 30 years earlier were used to calculate that 10-20 million of these $\mathrm{IgG}^{+}$memory B cells were present in the spleen, whereas less than $10^{5}$ could be detected in the blood. So, although these memory B cells represent just over $0.2 \%$ of all of the $\mathrm{IgG}^{+}$cells in the spleen and therefore make a discernable contribution to the splenic B-cell population, they only correspond to $0.07 \%$ of the $\operatorname{IgG}^{+}$cells in the blood ${ }^{84}$. Consequently, the identification and assessment of absolute numbers of specific memory B cells might be difficult to determine from blood samples.

Determinants of the B-cell pool. The progressive and unidirectional nature of B-cell development means that ageing should be associated with an increase in the number of plasma cells, but it is not clear whether this process is accompanied by an increase in the number of survival niches. The number of these niches is thought to be finite ${ }^{85-87}$ but might depend on age; age-related changes in the bone marrow could alter the number of survival niches, and access to these niches is known to regulate the size of the plasma-cell pool ${ }^{78}$. In old mice, an impaired ability of the aged bone marrow to support plasma-cell survival was proposed to account for the decreased number of long-lived bone marrow plasma cells ${ }^{88}$. Whether agerelated changes in the bone marrow alter plasma-cell number in older humans is unknown, as is how these changes could account for the increased prevalence of monoclonal gammopathy in older individuals ${ }^{89}$.

Determinants of the B-cell response. The failure of older individuals to produce an antibody response of the same magnitude as that from a young individual led to studies that aimed to determine whether this defect was due to the absence of a specific contributing cell type or a component of the response. Histological analysis of lymph nodes from human subjects has shown that the number and size of germinal centres are significantly decreased in older individuals (TABLE 1), and these changes are accompanied by an increase in the amount of adipose tissue $e^{90}$. These alterations suggest that there might be limitations to the proliferative phase of the immune response in the elderly. Indeed, studies examining mitogen-induced proliferation of T cells from older individuals show that these $\mathrm{T}$ cells have considerably less proliferative capacity in vitro compared with $\mathrm{T}$ cells from younger individuals ${ }^{91}$. B cells from old mice also show decreased proliferation in vitro compared with B cells from younger animals ${ }^{92}$, although this effect does not seem to be as marked as for T cells. A possible explanation for this might come from experiments looking at telomeres and telomerase, which have a central role in the replicative lifespan of cells. Comparison 


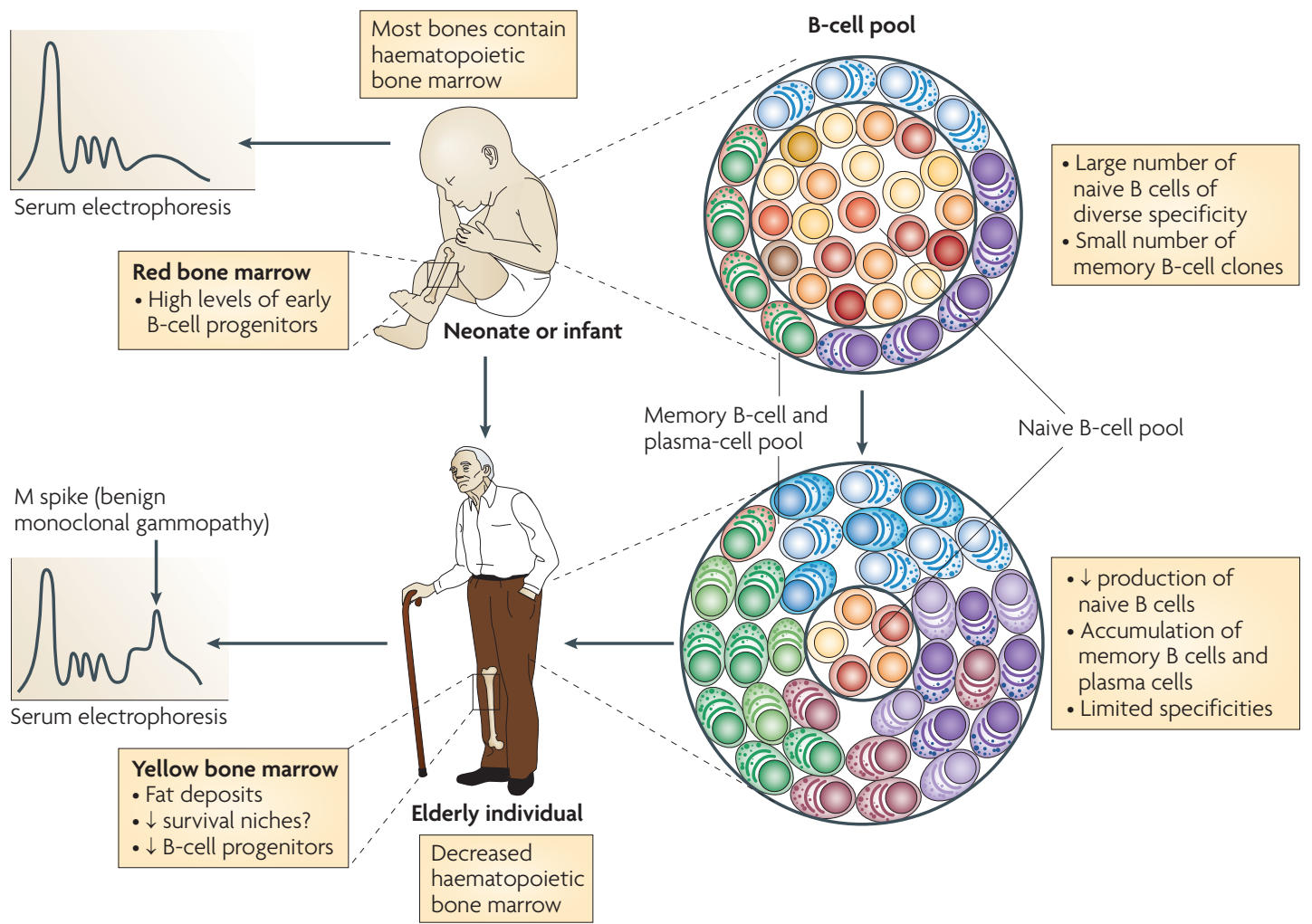

Figure 2 | Comparative changes in the B-cell pool. In early life, the haematopoietic bone marrow generates large numbers of pre-B cells and contains naive B cells of diverse specificities and a small number of memory B-cell clones. With age, the production of naive $B$ cells declines, contributing to the decrease in the capacity to respond to new antigens. Memory B cells and plasma cells of limited specificities accumulate, and occasional dysregulated plasma cells might produce monoclonal lgG (M spike); this might limit the access of new plasma cells to functional bone marrow niches. In addition, differences are observed between the bone marrow of neonates or infants and the elderly. Specifically, in early life, most bones contain marrow that has high levels of B-cell progenitors and is actively producing B cells. Age-related changes in the bone marrow, including the accumulation of fat deposits, are associated with the potential loss of specialized survival niches and a decrease in the number of $\mathrm{B}$-cell progenitors.

of naive and memory $\mathrm{CD} 4^{+} \mathrm{T}$ cells from the blood of healthy adult donors revealed that naive cells consistently have longer telomeres than memory cells (which have undergone extensive proliferation $)^{93}$. By contrast, no such consistent decline in telomere length was noted when naive and memory $\mathrm{B}$ cells were analysed ${ }^{94}$, despite the fact that B-cell differentiation is accompanied by considerable cell division. Lengthening of the telomeres through the induced expression of telomerase in the germinal centre during proliferation and differentiation might account for this observation ${ }^{94}$. Another study has shown that there does seem to be some age-related loss of telomere length in B cells, but that the rate of telomere shortening in B cells is significantly slower than that seen in $\mathrm{CD}^{+}{ }^{+} \mathrm{T}_{\text {cells }}{ }^{95}$.

Others have concentrated their studies on the role of FDCs and immune complexes in the age-related decrease in B-cell responses. Immune complexes on FDCs have been reported to stimulate B-cell proliferation in the germinal centre ${ }^{96}$. In addition, studies in mouse models showed that although the numbers of FDCs in young and old animals are not altered, there is a change in their functional ability. Specifically, FDCs from old mice have been shown to have a marked decrease (>90\%) in their expression of low-affinity Fc receptor for IgG (Fc $\gamma$ RII) compared with young animals, which has been proposed to contribute to the decline in the retention of immune complexes in the germinal centre ${ }^{97}$. This might be responsible for the decrease in number and size of germinal centres, and might contribute to the shorter duration of antibody responses that is observed in the elderly (TABLE 1).

An alternative explanation for the decrease in antibody responses with age might be related to the expression level of CD86 on B cells. Young animals that have been injected with a CD86-specific antibody have a decreased ability to form germinal centres and their B cells fail to undergo somatic mutation of immunoglobulins and to produce high-affinity antibody ${ }^{74}$. These results prompted the hypothesis that the decreased antibody responses in older individuals could be due to decreased expression of CD86 on their B cells. Initial experiments failed to support this notion, as germinal centre $B$ cells from old mice have a normal activation phenotype ${ }^{98}$. Further experiments sought to determine whether the defect was upstream of this process. One such early step is the interaction between CD40 on B cells or antigen-presenting cells and CD40L on $\mathrm{T}$ cells, which has been linked with the upregulation 
of expression of CD86 and, consequently, with successful B-cell responses ${ }^{99}$. Adoptive transfer of T cells from old animals to young hosts resulted in decreased B-cell proliferation and differentiation in response to antigen, whereas no difference in B-cell proliferation and differentiation was noted when young $\mathrm{T}$ cells were transferred to young and old hosts. These age-related defects might be due to the decreased level of expression of CD40L on the T cells from old animals ${ }^{100,101}$

Several studies indicate that older humans have the capacity to generate a diverse immunoglobulin repertoire and a similar range of immunoglobulin rearrangements as younger individuals ${ }^{102,103}$. Some studies suggest that although the quantity of antibody that is produced does not change greatly with age, the quality declines markedly, with older individuals producing fewer antigen-specific antibodies and a higher number of antigen-non-specific antibodies ${ }^{104}$. This limitation cannot be explained entirely by defects in the B-cell population; problems with other cell populations (such as FDCs and T cells) could also be implicated.

Changes in antibody responses in older individuals are associated with a decline in the production of naive $B$ cells and the accumulation of memory B cells. This increase in memory B cells is not paralleled by a similar increase in the number of plasma cells, which indicates that these two populations are not intimately linked or that there is a decrease in the number of accessible and functional plasma-cell survival niches in the bone marrow. The shift in the ratio of naive to memory B cells, which might be due in part to an intrinsic property of the stem cells of old animals ${ }^{105}$, provides a plausible explanation for the age-associated restriction in the B-cell repertoire. Evidence for a decline in the repertoire comes from recent studies of spectratype analysis of the immunoglobulin heavy-chain variable region complementaritydetermining region in DNA. These studies revealed a marked age-associated decrease in the B-cell repertoire that could involve clonal B-cell expansion in vivo ${ }^{106}$.
In summary, the two extremes of age share a similarly high vulnerability to common pathogens such as RSV, influenza virus and S. pneumoniae. Remarkably, they share similar difficulties in eliciting sustained protective antibody responses, and this essentially results from the influence of a limited number of non-B-cell determinants, including FDCs and bone marrow survival niches for plasma cells.

\section{Towards improved immunization strategies}

Circumventing the limitations of antibody responses to vaccination at the extremes of age is not trivial (TABLE 2). When high levels of neutralizing antibody are required for protection against pathogens in early life (such as RSV and influenza virus), new adjuvants and/or delivery systems that increase B-cell activation and plasma-cell differentiation will be required. When there is sufficient time for the induction of a memory response and its subsequent activation, the preferential differentiation pathway of early-life B cells towards memory B cells could benefit from 'early prime - later boost' immunization strategies. This implies defining the earliest age at which specific vaccine antigens can efficiently prime neonatal $\mathrm{B}$ and $\mathrm{T}$ cells of the appropriate phenotype. It also implies giving the final vaccine dose after a sufficiently long interval (3-4 months) for affinity maturation to occur. Defining strategies that elicit the appropriate balance between plasma cells and memory B cells, result in sustained antibody production and avoid vaccine interference by altered B- and/or T-cell responses ${ }^{50}$ will be the next challenge. Providing passive protection through maternal immunization ${ }^{107}$ is an attractive alternative for pathogens to which exposure occurs early, although addressing the issues of low acceptance and fear of litigation will be complicated.

In older individuals, increased doses of antigen or new adjuvants might enhance vaccine immunogenicity ${ }^{108}$. However, the main limiting factor is the ageassociated atrophy of the haematopoietic tissue and

\begin{tabular}{|c|c|}
\hline Mechanisms & Potential strategies \\
\hline \multicolumn{2}{|c|}{ Primary activation of naive antigen-specific B cells } \\
\hline Increasing recruitment of naive $B$ cells & $\begin{array}{l}\text { - Administering higher doses of vaccine antigen } \\
\text { - Increasing antigen retention by improving depot formulation } \\
\text { and antigen delivery systems } \\
\text { - Improving B-cell targeting with new antigen delivery systems } \\
\text { - Administering repeated primary immunization doses }\end{array}$ \\
\hline Increasing activation of naive B cells & - Developing new adjuvants, delivery systems and immunomodulators \\
\hline \multicolumn{2}{|l|}{ Enhancement of plasma-cell responses } \\
\hline Increasing B-cell activation & $\begin{array}{l}\text { - Administering higher doses of vaccine antigen } \\
\text { - Developing new adjuvants and immunomodulators }\end{array}$ \\
\hline Increasing plasma-cell differentiation & - Developing new adjuvants and immunomodulators \\
\hline Improving plasma-cell survival & - Improving access of plasma cells to functional bone marrow niches \\
\hline \multicolumn{2}{|l|}{ Enhancement of memory B-cell responses } \\
\hline Increasing memory B-cell differentiation & - Administering lower doses of vaccine antigen \\
\hline Recalling memory B-cell responses & $\begin{array}{l}\text { - Extending vaccine schedules (late boosters) } \\
\text { - Administering repeat booster doses }\end{array}$ \\
\hline
\end{tabular}


primary lymphoid organs. The accumulation of fat in the thymus and the bone marrow is associated with decreased production and export of new B and T cells, resulting in a peripheral lymphocyte pool that is shaped by previously encountered antigen. Decreased production of lymphocytes creates a system with fewer naive cells and, consequently, with increased vulnerability to pathogens that have not been encountered previously. In addition, the powerful homeostatic forces that maintain lymphocyte numbers through the proliferation of existing clones result in a greater number of cells that have reached their replicative limit, leaving older individuals vulnerable even to infections that have been successfully dealt with in the past. Reversal of thymic atrophy by castration ${ }^{109}$, the use of ligands for growth hormone receptors ${ }^{110}$ and IL-7 (REF. 111) have shown some success. Although these therapies have concentrated on T cells, they might also have an effect on B-cell development: older mice treated with IL-16 show a 1.6-fold increase in the number of pre-B cells compared with control animals ${ }^{112}$. How much of this might eventually apply to older humans remains to be determined.
1. Bryce, J., Boschi-Pinto, C., Shibuya, K. \& Black, R. E. WHO estimates of the causes of death in children. Lancet 365, 1147-1152 (2005).

2. Levy, O. Innate immunity of the newborn: basic mechanisms and clinical correlates. Nature Rev. Immunol. 7, 379-390 (2007)

3. Zinkernagel, R. M. Maternal antibodies, childhood infections, and autoimmune diseases. N. Engl. J. Med 345, 1331-1335 (2001)

4. Yoshikawa, T. T. Epidemiology and unique aspects of aging and infectious diseases. Clin. Infect. Dis. 30, 931-933 (2000)

5. Yoshikawa, T. T. Perspective: aging and infectious diseases: past, present, and future. J. Infect. Dis. 176 1053-1057 (1997).

6. Thompson, W. W. et al. Mortality associated with influenza and respiratory syncytial virus in the United States. JAMA 289, 179-186 (2003).

7. Gardner, P. \& Pabbatireddy, S. Vaccines for women age 50 and older. Emerg. Infect. Dis. 10, 1990-1995 (2004).

8. Streilein, J. W. Neonatal tolerance: towards an immunogenetic definition of self. Immunol. Rev. 46, 123-146 (1979)

9. Siegrist, C. Neonatal and early life vaccinology. Vaccine 19, 3331-3346 (2001)

10. Adkins, B., Leclerc, C. \& Marshall-Clarke, S. Neonatal adaptive immunity comes of age. Nature Rev. Immunol. 4, 553-564 (2004).

11. Hannoun, C., Megas, F. \& Piercy, J. Immunogenicity and protective efficacy of influenza vaccination. Virus Res. 103, 133-138 (2004).

12. Looney, R. J. et al. Hepatitis B immunization of healthy elderly adults: relationship between naiv $\mathrm{CD}^{+} \mathrm{T}$ cells and primary immune response and evaluation of GM-CSF as an adjuvant. J. Clin. Immunol. 21, 30-36 (2001).

13. Pollard, A., Perrett, K. P. \& Beverley, P. C. Maintaining protection against invasive bacteria with proteinpolysaccharide conjugate vaccines. Nature Rev. Immunol. 13 Feb 2009 (doi: 10.1038/nri2494).

14. Wright, P. F. et al. Evaluation of a live, cold-passaged, temperature-sensitive, respiratory syncytial virus vaccine candidate in infancy. J. Infect. Dis. 182, 1331-1342 (2000).

15. Halsey, N. \& Galazka, A. The efficacy of DPT and oral poliomyelitis immunization schedules initiated from birth to 12 weeks of age. Bull. World Health Organ 63, 1151-1169 (1985)

16. Einhorn, M. S., Weinberg, G. A., Anderson, E. L., Granoff, P. D. \& Granoff, D. M. Immunogenicity in infants of Haemophilus influenzae type B polysaccharide in a conjugate vaccine with Neisseria meningitidis outer-membrane protein. Lancet 2, 299-302 (1986).

17. Booy, R. et al. Immunogenicity of combined diphtheria, tetanus, and pertussis vaccine given at 2 , 3 , and 4 months versus 3,5 , and 9 months of age. Lancet 339, 507-510 (1992)

18. Tiru, M., Hallander, H. O., Gustafsson, L., Storsaeter, J. \& Olin, P. Diphtheria antitoxin response to DTP vaccines used in Swedish pertussis vaccine trials, persistence and projection for timing of booster. Vaccine 18, 2295-2306 (2000).

19. Olin, P., Hallander, H. O., Gustafsson, L., Barreto, L. \& Podda, A. Measuring protection; a case study of pertussis vaccines - Swedish Trial II: secondary nonrandomized comparisons between two schedules of infant vaccination. Dev. Biol. Stand. 95, 211-220 (1998).

20. Johnson, C. E. et al. Measles vaccine immunogenicity in 6-versus 15-month-old infants born to mothers in the measles vaccine era. Pediatrics $93,939-944$ (1994).
21. Gans, H. A. et al. Deficiency of the humoral immune response to measles vaccine in infants immunized at age 6 months. JAMA 280, 527-532 (1998). This report shows the slow acquisition of the antibody-response capacity: only $36 \%$ of 6-month-old infants have seroprotective neutralizing antibody titres of 120 or higher after measles vaccination, compared with $100 \%$ of 9-month-old infants.

22. Letson, G. W. et al. Effect of maternal antibody on immunogenicity of hepatitis $A$ vaccine in infants. J. Pediatr. 144, 327-332 (2004)

This study shows that the slow acquisition of the antibody response capacity also occurs in response to potent non-live vaccines, such as hepatitis $A$.

23. Giuliano, M. et al. Antibody responses and persistence in the two years after immunization with two acellular vaccines and one whole-cell vaccine against pertussis. J. Pediatr. 132, 983-988 (1998)

24. Whittle, H. C. et al. Effect of subclinical infection on maintaining immunity against measles in vaccinated children in West Africa. Lancet 353, 98-102 (1999)

25. Trotter, C. L., Andrews, N. J., Kaczmarski, E. B., Miller, E. \& Ramsay, M. E. Effectiveness of meningococcal serogroup $C$ conjugate vaccine 4 years after introduction. Lancet 364, 365-367 (2004).

26. Roduit, C. et al. Immunogenicity and protective efficacy of neonatal vaccination against Bordetella pertussis in a murine model: evidence for early control of pertussis. Infect. Immun. 70, 3521-3528 (2002).

27. Kaur, K., Chowdhury, S., Greenspan, N. S. \& Schreiber, J. R. Decreased expression of tumor necrosis factor family receptors involved in humoral immune responses in preterm neonates. Blood $\mathbf{1 1 0}$ 2948-2954 (2007).

28. Timens, W., Boes, A., Rozeboom-Uiterwijk, T. \& Poppema, S. Immaturity of the human splenic marginal zone in infancy. Possible contribution to the deficient infant immune response. J. Immunol. 143, 3200-3206 (1989).

This seminal work identifies crucial maturation stages for the development of the marginal zone of the spleen.

29. Griffioen, A. W., Rijkers, G. T., Janssens-Korpela, P. \& Zegers, B. J. Pneumococcal polysaccharides complexed with $\mathrm{C} 3 \mathrm{~d}$ bind to human B lymphocytes via complement receptor type 2. Infect. Immun. 59, 1839-1845 (1991).

30. Kanswal, S., Katsenelson, N., Selvapandiyan, A. Bram, R. J. \& Akkoyunlu, M. Deficient TACl expression on $B$ lymphocytes of newborn mice leads to defective Ig secretion in response to BAFF or APRIL. J. Immunol. 181, 976-990 (2008)

31. Siegrist, C. A. Mechanisms by which maternal antibodies influence infant vaccine responses: review of hypotheses and definition of main determinants. Vaccine 21, 3406-3412 (2003).

32. Johnston, R. B. Jr, Altenburger, K. M., Atkinson, A. W. Jr \& Curry, R. H. Complement in the newborn infant. Pediatrics 64, 781-786 (1979).

33. Pihlgren, M. et al. Influence of complement C3 amount on IgG responses in early life: immunization with C3bconjugated antigen increases murine neonatal antibody responses. Vaccine 23, 329-335 (2004).

34. Landers, C. D., Chelvarajan, R. L. \& Bondada, S. The role of $B$ cells and accessory cells in the neonatal response to TI-2 antigens. Immunol. Res. 31, 25-36 (2005).

35. Pihlgren, M. et al. Unresponsiveness to lymphoidmediated signals at the neonatal follicular dendritic cell precursor level contributes to delayed germinal center induction and limitations of neonatal antibody responses to T-dependent antigens. J. Immunol. 170 2824-2832 (2003).
This work provides a mechanistic explanation for the delayed and limited antibody responses in early life by identifying the slow postnatal maturation of FDCs as a crucial factor that decreases germinal centre responses.

36. Kruschinski, C., Zidan, M., Debertin, A. S., von Horsten, S. \& Pabst, R. Age-dependent development of the splenic marginal zone in human infants is associated with different causes of death. Hum. Pathol. 35, 113-121 (2004).

37. Blanchard Rohner, G. et al. The magnitude of the antibody and memory B cell responses during priming with a protein-polysaccharide conjugate vaccine in human infants is associated with the persistence of antibody and the intensity of booster response. J. Immunol. 180, 2165-2173 (2008).

38. Kovarik, J. et al. Adjuvant effects of $\mathrm{CpG}$ oligodeoxynucleotides on responses against T-independent type 2 antigens. Immunology 102, 67-76 (2001)

39. Pihlgren, M. et al. Delayed and deficient establishment of the long-term bone marrow plasma cell pool during early life. Eur. J. Immunol. 31, 939-946 (2001)

40. Pihlgren, M. et al. Reduced ability of neonatal and early-life bone marrow stromal cells to support plasmablast survival. J. Immunol. 176, 165-172 (2006).

41. Belnoue, E. et al. APRIL is critical for plasmablast survival in the bone marrow and poorly expressed by early-life bone marrow stromal cells. Blood 111 , 2755-2764 (2008).

This work identifies a unique pro-survival effect of APRIL on antigen-specific plasmablasts and shows that its limited production by bone marrow stromal cells is associated with the defective establishment of the plasma-cell pool.

42. Newport, M. J. et al. Genetic regulation of immune responses to vaccines in early life. Genes Immun. 5, 122-129 (2004).

43. Marchant, A. et al. Predominant influence of environmental determinants on the persistence and avidity maturation of antibody responses to vaccines in infants. J. Infect. Dis. 193, 1598-1605 (2006).

44. Dong, D. X. et al. Immunization of neonates with trivalent oral poliomyelitis vaccine (Sabin). Bull. World Health Organ. 64, 853-860 (1986).

45. Pichichero, M. E. et al. Impact of a birth dose of hepatitis $B$ vaccine on the reactogenicity and immunogenicity of diphtheria-tetanus-acellular pertussis-hepatitis B-inactivated poliovirusHaemophilus influenzae type b combination vaccination Pediatr. Infect. Dis. J. 21, 854-859 (2002).

46. Belloni, C. et al. Immunogenicity of a three-component acellular pertussis vaccine administered at birth Pediatrics 111, 1042-1045 (2003).

47. Knuf, M. et al. Neonatal vaccination with an acellular pertussis vaccine accelerates the acquisition of pertussis antibodies in infants. J. Pediatr. 152 655-660 (2008).

48. Dengrove, J. et al. IgG and IgG subclass specific antibody responses to diphtheria and tetanus toxoids in newborns and infants given DTP immunization. Pediatr. Res. 20, 735-739 (1986).

49. Halasa, N. B., O'Shea, A., Shi, J. R., LaFleur, B. J. ¿ Edwards, K. M. Poor immune responses to a birth dose of diphtheria, tetanus, and acellular pertussis vaccine. J. Pediatr. 153, 327-332 (2008).

50. Siegrist, C. A. Blame vaccine interference, not neonatal immunization, for suboptimal responses after neonatal diphtheria, tetanus, and acellular pertussis immunization. J. Pediatr. 153, 305-307 (2008). 
51. Ridings, J., Dinan, L., Williams, R., Roberton, D. \& Zola, H. Somatic mutation of immunoglobulin $V_{H} 6$ genes in human infants. Clin. Exp. Immunol. 114, 33-39 (1998)

52. Lucas, A. H., Azmi, F. H., Mink, C. M. \& Granoff, D. M. Age-dependent $\mathrm{V}$ region expression in the human antibody response to the Haemophilus influenzae type b polysaccharide. J. Immunol. 150, 2056-2061 (1993).

53. Schallert, N. et al. Generation of adult-like antibody avidity profiles after early-life immunization with protein vaccines. Eur J. Immunol. 32, 752-760 (2002).

54 Hetherington, S. V. \& Rutkowski, A. F. Antibody affinity in infants after immunization with conjugated capsular polysaccharide from Haemophilus influenzae type b. J. Infect. Dis. 162, 1185-1188 (1990).

55. Weitkamp, J. H et al. Infant and adult human B cell responses to rotavirus share common immunodominant variable gene repertoires. J. Immunol. 171, 4680-4688 (2003).

56. Zemlin, M. et al. The postnatal maturation of the immunoglobulin heavy chain IgG repertoire in human preterm neonates is slower than in term neonates. J. Immunol. 178, 1180-1188 (2007).

57. Kolar, G. R., Yokota, T., Rossi, M. I., Nath, S. K. $\overline{1}$ Capra, J. D. Human fetal, cord blood, and adult lymphocyte progenitors have similar potential for generating $\mathrm{B}$ cells with a diverse immunoglobulin repertoire. Blood 104, 2981-2987 (2004).

58. Nair, N. et al. Age-dependent differences in IgC isotype and avidity induced by measles vaccine received during the first year of life. J. Infect. Dis. 196, 1339-1345 (2007)

59. Smith, K. G., Light, A., Nossal, G. J. \& Tarlinton, D. M The extent of affinity maturation differs between the memory and antibody-forming cell compartments in the primary immune response. EMBO J. 16 2996-3006 (1997).

60. Gatto, D. et al. Complement receptors regulate differentiation of bone marrow plasma cell precursors expressing transcription factors Blimp-1 and XBP-1. J. Exp. Med. 201, 993-1005 (2005)

61. Bryant, V. L. et al. Cytokine-mediated regulation of human B cell differentiation into Ig-secreting cells: predominant role of IL-21 produced by CXCR5 T follicular helper cells. J. Immunol. 179, 8180-8190 (2007).

62. Benson, M. J., Erickson, L. D., Gleeson, M. W. \& Noelle, R. J. Affinity of antigen encounter and other early B-cell signals determine B-cell fate. Curr. Opin. Immunol. 19, 275-280 (2007).

63. Latz, E., Franko, J., Golenbock, D. T. \& Schreiber, J. R. Haemophilus influenzae type b-outer membrane protein complex glycoconjugate vaccine induces cytokine production by engaging human toll-like receptor 2 (TLR2) and requires the presence of TLR2 for optimal immunogenicity. J. Immunol. 172, 2431-2438 (2004)

64. Decker, M. D., Edwards, K. M., Bradley, R. \& Palmer, P. Comparative trial in infants of four conjugate Haemophilus influenzae type b vaccines. J. Pediatr. 120, 184-189 (1992).

65. Borrow, R. et al. Immunogenicity of, and immunologic memory to, a reduced primary schedule of meningococcal $\mathrm{C}$-tetanus toxoid conjugate vaccine in infants in the United Kingdom. Infect. Immun. 71 5549-5555 (2003).

66. Samandari, T. et al. Differences in response to a hepatitis B vaccine booster dose among Alaskan children and adolescents vaccinated during infancy. Pediatrics 120, e373-e381 (2007)

67. Lu, C. Y. et al. Humoral and cellular immune responses to a hepatitis B vaccine booster 15-18 years after neonatal immunization. J. Infect. Dis. 197 1419-1426 (2008)

68. Amanna, I. J., Carlson, N. E. \& Slifka, M. K Duration of humoral immunity to common viral and vaccine antigens. N. Engl. J. Med. 357, 1903-1915 (2007).

An excellent paper that measures the duration of antiviral antibody responses and shows that for most of the responses tested there was no correlation between peripheral memory B-cell numbers and antibody levels.

69. Ochsenbein, A. F. et al. Protective long-term antibody memory by antigen-driven and $\mathrm{T}$ help-dependent differentiation of long-lived memory B cells to shortlived plasma cells independent of secondary lymphoid organs. Proc. Natl Acad. Sci. USA 97, 13263-13268 (2000).

70. Goodwin, K., Viboud, C. \& Simonsen, L. Antibody response to influenza vaccination in the elderly: a quantitative review. Vaccine 24, 1159-1169 (2006).
71. Melegaro, A. \& Edmunds, W. J. The 23-valent pneumococcal polysaccharide vaccine. Part I. Efficacy of PPV in the elderly: a comparison of meta-analyses. Eur. J. Epidemiol. 19, 353-363 (2004).

72. Weinberger, B., Herndler-Brandstetter, D. Schwanninger, A., Weiskopf, D. \& GrubeckLoebenstein, B. Biology of immune responses to vaccines in elderly persons. Clin. Infect. Dis. 46, 1078-1084 (2008)

73. Hallgren, H. M., Buckley, C. E. III, Gilbertsen, V. A. \& Yunis, E. J. Lymphocyte phytohemagglutinin responsiveness, immunoglobulins and autoantibodies in aging humans. J. Immunol. 111, 1101-1107 (1973)

74. Weksler, M. E. \& Szabo, P. The effect of age on the B-cell repertoire. J. Clin. Immunol. 20, 240-249 (2000).

75. Welch, M. J., Fong, S., Vaughan, J. \& Carson, D. Increased frequency of rheumatoid factor precursor $B$ lymphocytes after immunization of normal adults with tetanus toxoid. Clin. Exp. Immunol. 51 299-304 (1983).

76. Mariotti, S. et al. Thyroid and other organ-specific autoantibodies in healthy centenarians. Lancet 339 1506-1508 (1992).

77. Kyle, R. A et al. Prevalence of monoclonal gammopathy of undetermined significance. N. Engl. J. Med. 354, 1362-1369 (2006)

78. Stoop, J. W., Zegers, B. J., Sander, P. C. \& Ballieux, R. E. Serum immunoglobulin levels in healthy children and adults. Clin. Exp. Immunol. 4, 101-112 (1969).

79. Kirkland, J. L., Tchkonia, T., Pirtskhalava, T., Han, J. \& Karagiannides, I. Adipogenesis and aging: does aging make fat go MAD? Exp. Gerontol. 37, 757-767 (2002)

80. Johnson, S. A. \& Cambier, J. C. Ageing, autoimmunity and arthritis: senescence of the B cell compartment implications for humoral immunity. Arthritis Res. Ther. 6, 131-139 (2004)

81. Bohnhorst, J. O., Bjorgan, M. B., Thoen, J. E. Natvig, J. B. \& Thompson, K. M. Bm1-Bm5 classification of peripheral blood $B$ cells reveals circulating germinal center founder cells in healthy individuals and disturbance in the $\mathrm{B}$ cell subpopulations in patients with primary Sjogren's syndrome. J. Immunol. 167, 3610-3618 (2001).

82. Wei, C. et al. A new population of cells lacking expression of $\mathrm{CD} 27$ represents a notable component of the B cell memory compartment in systemic lupus erythematosus. J. Immunol. 178, 6624-6633 (2007).

83. Sanz, I., Wei, C., Lee, F. E. \& Anolik, J. Phenotypic and functional heterogeneity of human memory B cells. Semin. Immunol. 20, 67-82 (2008).

84. Mamani-Matsuda, $M$ et al. The human spleen is a major reservoir for long-lived vaccinia virus-specific memory B cells. Blood 111, 4653-4659 (2008) This paper reports the differential distribution of memory B cells between blood and spleen, and the lack of any significant correlation between specific memory B cells in the blood and the serum titres of their specific antibody.

85. Fairfax, K. A., Kallies, A., Nutt, S. L. \& Tarlinton, D. M. Plasma cell development: from B-cell subsets to longterm survival niches. Semin. Immunol. 20, 49-58 (2008)

86. Tarlinton, D., Radbruch, A., Hiepe, F. \& Dorner, T. Plasma cell differentiation and survival. Curr. Opin. Immunol. 20, 162-169 (2008)

87. Radbruch, A. et al. Competence and competition: the challenge of becoming a long-lived plasma cell. Nature Rev. Immunol. 6, 741-750 (2006).

88. Han, S. et al. Enhanced differentiation of splenic plasma cells but diminished long-lived high-affinity bone marrow plasma cells in aged mice. J. Immunol. 170, 1267-1273 (2003).

89. Kyle, R. A. \& Rajkumar, S. V. Monoclonal gammopathy of undetermined significance. Br. J. Haematol. 134 573-589 (2006)

90. Luscieti, P., Hubschmid, T., Cottier, H., Hess, M. W. \& Sobin, L. H. Human lymph node morphology as a function of age and site. J. Clin. Pathol. 33, 454-46 (1980)

91. Pawelec, G. et al T cells and aging, January 2002 update. Front. Biosci. 7, d1056-d1183 (2002).

92. Frasca, D., Nguyen, D., Riley, R. L. \& Blomberg, B. B. Effects of aging on proliferation and E47 transcription factor activity induced by different stimuli in murine splenic B cells. Mech. Ageing Dev. 124, 361-369 (2003)

93. Weng, N. P., Levine, B. L., June, C. H. \& Hodes, R. J. Human naive and memory $T$ lymphocytes differ in telomeric length and replicative potential. Proc. Nat Acad. Sci. USA 92, 11091-11094 (1995).

94. Weng, N. P., Granger, L. \& Hodes, R. J. Telomere lengthening and telomerase activation during human
B cell differentiation Proc Natl Acad Sci. USA 94 10827-10832 (1997)

95. Son, N. H., Murray, S., Yanovski, J., Hodes, R. J. \& Weng, N. Lineage-specific telomere shortening and unaltered capacity for telomerase expression in human $\mathrm{T}$ and B lymphocytes with age. J. Immunol. 165, 1191-1196 (2000)

96. Aydar, Y., Wu, J., Song, J., Szakal, A. K. \& Tew, J. G FcyRll expression on follicular dendritic cells and immunoreceptor tyrosine-based inhibition motif signaling in B cells. Eur. J. Immunol. 34, 98-107 (2004).

97. Aydar, Y., Balogh, P., Tew, J. G. \& Szakal, A. K. Follicular dendritic cells in aging, a "bottle-neck" in the humoral immune response. Ageing Res. Rev. 3 , 15-29 (2004).

98. Rogerson, B. J., Harris, D. P., Swain, S. L. \& Burgess, D. O Germinal center B cells in Peyer's patches of aged mice exhibit a normal activation phenotype and highly mutated IgM genes. Mech Ageing Dev. 124, 155-165 (2003).

99. Lumsden, J. M., Williams, J. A. \& Hodes, R. J. Differential requirements for expression of CD80/86 and CD40 on B cells for T-dependent antibody responses in vivo. J. Immunol. 170, 781-787 (2003)

100. Eaton, S. M., Burns, E. M., Kusser, K., Randall, T. D. \& Haynes, L. Age-related defects in CD4 T cell cognate helper function lead to reductions in humoral responses. J. Exp. Med. 200, 1613-1622 (2004).

101. Haynes, L. \& Eaton, S. M. The effect of age on the cognate function of $\mathrm{CD}^{+}{ }^{+}$T cells. Immunol. Rev. 205 , 220-228 (2005)

102. Kolar, G. R., Mehta, D., Wilson, P. C. \& Capra, J. D. Diversity of the Ig repertoire is maintained with age in spite of reduced germinal centre cells in human tonsil lymphoid tissue. Scand. J. Immunol. 64, 314-324 (2006).

103. Banerjee, M., Mehr, R., Belelovsky, A., Spencer, J. \& Dunn-Walters, D. K. Age- and tissue-specific differences in human germinal center $\mathrm{B}$ cell selection revealed by analysis of IgVH gene hypermutation and lineage trees. Eur. J. Immunol. 32, 1947-1957 (2002).

104. Howard, W. A., Gibson, K. L. \& Dunn-Walters, D. K. Antibody quality in old age. Rejuvenation Res. 9, 117-125 (2006)

105. Guerrettaz, L. M., Johnson, S. A. \& Cambier, J. C. Acquired hematopoietic stem cell defects determine B-cell repertoire changes associated with aging. Proc. Natl Acad. Sci. USA 105, 11898-11902 (2008)

106. Gibson, K. L. et al. B-cell diversity decreases in old age and is correlated with poor health status. Aging Cell 8, 18-25 (2009)

A spectratype analysis across the complementaritydetermining region 3 in individuals of different ages that shows the decline in the B-cell repertoire in older individuals and at the same time maps their health status on the analysis, thereby linking frailty with repertoire distortion.

107. Healy, C. M. \& Baker, C. J. Maternal immunization. Pediatr. Infect. Dis. J. 26, 945-948 (2007).

108. De Bruijn, l. et al. Antibody induction by virosomal, MF59-adjuvanted, or conventional influenza vaccines in the elderly. Vaccine 26, 119-127 (2007).

109. Sutherland, J. S. et al. Activation of thymic regeneration in mice and humans following androgen blockade. J. Immunol. 175, 2741-2753 (2005).

110. Dixit, V. D. et al. Ghrelin promotes thymopoiesis during aging. J. Clin. Invest. 117, 2778-2790 (2007)

11 Aspinall, R. et al. Old rhesus macaques treated with interleukin-7 show increased TREC levels and respond well to influenza vaccination. Rejuvenation Res. 10 5-18 (2007)

112. Szabo, P. et al. Maturation of B cell precursors is impaired in thymic-deprived nude and old mice. J. Immunol. 161, 2248-2253 (1998).

\section{Acknowledgements}

The authors are grateful to all who participated in or supported their research projects.

DATABASES

Entrez Gene: http://www.ncbi.nlm.nih.gov/entrez/query.

fcgi? db=gene

APRIL $|\underline{B A F F}| \underline{C D 27}|\underline{C D 40}| \underline{C D 40 L}|\underline{C D 80}| \underline{C D 86}$

FURTHER INFORMATION

Claire-Anne Siegrist's homepage: http://pathology.unige. ch/group-siegrist.htm

World Health Organization (WHO) health statistics: http://www.who.int/whosis/whostat/2008/en/index.htm ALL LINKS ARE ACTIVE IN THE ONLINE PDF 